\title{
Valoración económica del secuestro y almacenamiento de carbono en la puna seca del suroeste del Perú
}

\author{
Economic valuation of carbon capture and storage \\ in the puna dry of southwestern Peru
}

\author{
César E Medina ${ }^{a *}$, Yasmy K Medina ${ }^{a}$, Edwin F Bocardo ${ }^{a}$

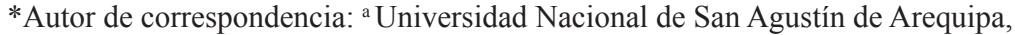 \\ Facultad de Ciencias Biológicas, Departamento Académico de Biología, Av. Alcides Carrión s/n., \\ Arequipa, Perú, tel.: 0051-940393978, cmedinap@unsa.edu.pe
}

\begin{abstract}
SUMMARY
In the face of a global warming scenario, carbon capture and storage has become an international strategy to mitigate the effect of greenhouse gases. Oceans and Tropical forests are considered the largest carbon sinks in the world, however a few studies have evaluated the relative importance of other plant formations as carbon sinks in the Neotropical Region. This study documents the economic value of carbon capture and storage by five plant formations representative in the Puna Seca of southwestern Peru. Sampling plots were established to estimate the biomass contained in seedlings of Pajonal, Tolar, Bofedal, Yaretal and Queñual, in Salinas y Aguada Blanca National Reserve (Arequipa, Peru). Later, carbon stored and carbon captured as $\mathrm{CO}_{2}$ was estimated. The Peruvian average carbon exchange price per ton of $\mathrm{CO}_{2}$ was used for the economic evaluation of environmental services. It was found that the Reserve contains at least 13,507,104.16 equivalent metric tons of $\mathrm{CO}_{2}$ worth U\$D 86,310,395.58. Bofedal was the main vegetal formation that contributed in these values $(52.48 \%)$, in spite of being little represented in the total area of the Reserve (2.58 \%). It is expected that the economic value obtained will serve as a frame of reference in decision-making for various purposes (environmental awareness, environmental management instruments, national accounting, among others).
\end{abstract}

Key words: Arequipa, biomass, $\mathrm{CO}_{2}$ sink, ecosystem service, Salinas y Aguada Blanca National Reserve.

\section{RESUMEN}

Ante un panorama de calentamiento global, la captura y secuestro de carbono se ha convertido en una estrategia internacional para mitigar el efecto de los gases invernadero. Los océanos y los bosques tropicales son considerados como los mayores sumideros de carbono en el mundo, sin embargo, pocos estudios han evaluado la importancia relativa de otras formaciones vegetales como sumideros de carbono en la región Neotropical. El presente estudio documenta el valor económico de la captura y secuestro de carbono por cinco formaciones vegetales representativas de la Puna Seca del suroeste del Perú. Se establecieron parcelas de muestreo para estimar la biomasa contenida en plántulas del Pajonal, Tolar, Bofedal, Yaretal y Queñual, en la Reserva Nacional Salinas y Aguada Blanca (Arequipa, Perú), posteriormente, se estimó el carbono almacenado y el carbono capturado como $\mathrm{CO}_{2}$. Se utilizó el precio promedio de la tonelada de $\mathrm{CO}_{2}$ equivalente, establecido en el mercado peruano, para obtener el valor económico del servicio ambiental. Como resultados, se observó que la Reserva contiene al menos 13.507.104,16 toneladas métricas de $\mathrm{CO}_{2}$ equivalente, valoradas en USD 86.310.395,58. El Bofedal fue la principal formación vegetal que contribuyó en dichos valores (52,48 \%), a pesar de estar poco representado en la superficie total de la Reserva (2,58 \%). Se espera que el valor económico obtenido sirva de marco de referencia en la toma de decisiones para fines diversos (concientización ambiental, instrumentos de gestión ambiental, contabilidad nacional, entre otros).

Palabras clave: Arequipa, biomasa, Reserva Nacional de Salinas y Aguada Blanca, servicio ecosistémico, sumidero de $\mathrm{CO}_{2}$.

\section{INTRODUCCIÓN}

Uno de los problemas ambientales más severos al que nos enfrentamos en el presente siglo es el Cambio Climático, el cual se debe al incremento en las emisiones antropogénicas de gases de efecto invernadero, tales como dióxido de carbono $\left(\mathrm{CO}_{2}\right)$, monóxido de carbono, clorofluorocarbonados, óxidos de nitrógeno y metano, siendo el $\mathrm{CO}_{2}$ uno de los gases más importantes por las grandes cantidades en las que se emiten a la atmósfera (Phillips et al. 2017). La preocupación mundial por mitigar el efecto de dichos gases ha dado lugar a una política internacional dirigida a entender los procesos de generación y absorción de ellos, buscando mantener el aumento 
de la temperatura media mundial muy por debajo de $2{ }^{\circ} \mathrm{C}$ con respecto a los niveles preindustriales, y proseguir los esfuerzos para limitar ese aumento de la temperatura a $1,5^{\circ} \mathrm{C}$ con respecto a los niveles preindustriales (Trinidad y Ortiz 2019). Esto ha permitido reconocer la importancia de los ecosistemas terrestres y, en particular, el papel que tiene la vegetación para captar el $\mathrm{CO}_{2}$ atmosférico por medio de la fotosíntesis, incorporándolo a las estructuras vegetales, y mitigando a largo plazo el cambio climático (Sullivan et al. 2017).

La medición y el monitoreo de carbono en ecosistemas de alta montaña se ha tornado un importante tópico de investigación en los años recientes, siendo importante su cuantificación para disponer de datos empíricos en las negociaciones para reducir las emisiones de gases efecto invernadero asociadas a deforestación y degradación forestal (REDD+). La fijación de un precio al carbono viene siendo una política climática cada vez más prioritaria en los países de América Latina (Trinidad y Ortiz 2019). El Perú, como país miembro de la Convención Marco de las Naciones Unidas para el Cambio Climático y comprometido con la reducción gases de efecto invernadero a través del Protocolo de Kioto, ha adoptado una serie de instrumentos económicos y de mercado (Bonos de carbono) para mitigar las emisiones de gases de efecto invernadero (Phillips et al. 2017).

A nivel regional, el Perú se encuentra en una categoría de alto riesgo a los impactos asociados al cambio climático (Trinidad y Ortiz 2019). Dicha ubicación es de singular importancia debido a los procesos de deforestación, tala ilegal, cambio en el uso de suelo e incendios forestales vienen originado cinco puntos focales (Hotspot) de deforestación en la Amazonía del Perú, ocasionando la pérdida de 59 millones de toneladas métricas de carbono entre 2013 a 2017 (Martel y Cairampoma 2012).

Estudios sobre la valoración del servicio ecosistémico de captura y almacenamiento de $\mathrm{CO}_{2}$ en Perú han sido desarrollados principalmente en el Amazonía (Glave y Pizarro 2001, Martel y Cairampoma 2012). En cambio, estudios en regiones altoandinas son escasos, los cuales han sido realizados de forma particular en formaciones vegetales como Pajonal, Bofedal o Queñual, haciendo difícil la incorporación de su valor económico en la toma de decisiones para el manejo de recursos naturales altoandinos (Glave y Pizarro 2001, Gibbon et al. 2010, Medrano et al. 2012, Vásquez et al. 2014, Crispin 2015, Morales 2015, Limache 2016, Flores 2017, Sarcca 2017).

Por consiguiente, la pregunta de investigación que fundamenta este estudio es cuál es la importancia relativa del Pajonal, Tolar, Bofedal, Yaretal y Queñual en la puna seca del suroeste del Perú, a base del valor económico que representan por el servicio de secuestro y almacenamiento de $\mathrm{CO}_{2}$. Se planteó como objetivo estimar el valor económico asociado al secuestro y almacenamiento de $\mathrm{CO}_{2}$ en los principales tipos de vegetación de la Reserva Nacional Salinas y Aguada Blanca (Arequipa, Perú), considerando como hipótesis que el valor económico del servicio ecosistémico debería variar según la formación vegetal y la superficie que esta ocupa en la RNSAB. Para cumplir con lo propuesto, se estimó la biomasa y carbono capturado en parcelas de muestreo y se utilizó el método de precio de mercado, aplicando el valor asignado por tonelada de $\mathrm{CO}_{2}$ equivalente implementado en el Estado Peruano.

\section{MÉTODOS}

Área de estudio. La Reserva Nacional de Salinas y Aguada Blanca (RNSAB) es una de las principales Áreas Naturales Protegidas del Perú que resguarda una muestra representativa de la puna seca en el suroeste del país. Se ubica en los departamentos de Arequipa y Moquegua, ocupando un área de 366.936 ha, en un rango altitudinal que va de los 2.800 a más de $6.000 \mathrm{~m}$ de altitud (figura 1).

El clima de toda la RNSAB está dominado por dos condiciones, la altura y la sequedad, que le confieren un clima de montaña notablemente árido. La precipitación en el área varía entre 80 a $1.000 \mathrm{~mm}$, presentándose marcada estacionalidad lluviosa, mayormente restringida a los meses de verano, con sequías frecuentes. Hacia el oeste de la Reserva llueve menos de $250 \mathrm{~mm}$, por lo que se le podría considerar como desértico, mientras que la mayor parte del área es semidesértica. La fuerte insolación es característica de ambientes montañosos. La humedad relativa es el principal factor que limita la distribución de las plantas y los animales, llegando a alcanzar un $60 \%$ en promedio. La temperatura promedio en la zona varía entre los 2 y $8^{\circ} \mathrm{C}$, con mínimas de hasta $-10^{\circ} \mathrm{C}$ (Zeballos et al. 2010).

Desde el punto de vista geomorfológico, es una planicie elevada (meseta), por sobre la cual se encuentran estructuras volcánicas que son parte de la cordillera volcánica del sur peruano. La geomorfología actual del área es el resultado del efecto combinado de fuerzas endógenas y exógenas que se desarrollaron durante el terciario y el cuaternario gracias a procesos morfodinámicos que en sus fases agradacionales corresponden al dimorfismo vertical $\mathrm{y}$ al vulcanismo Plio-Pleistoceno, y en sus fases gradacionales a la acción erosiva de períodos pluvio glaciales (Zeballos et al. 2010).

La flora de la RNASB consta de más de 463 especies de plantas vasculares, siendo las Asteraceae y Poaceae las familias con mayor número de especies; mientras que, los vertebrados suman 207 especies, conformados por 37 mamíferos (34 nativos y tres introducidos en estado silvestre), 158 aves, cinco reptiles, cuatro anfibios y tres peces (dos nativos y uno introducido) (Zeballos et al. 2010).

Las principales formaciones vegetales que ocurren en la RNSAB, según su representatividad y estado de conservación, son: Pajonal, dominado por Stipa spp. Linnaeus y Festuca orthophylla Pilg. (con una superficie de 317.134,84 ha); Tolar, dominado por Parastrephia spp. Nutt. y Baccharis spp. Linnaeus (48.623,80 ha); Bofedal, 


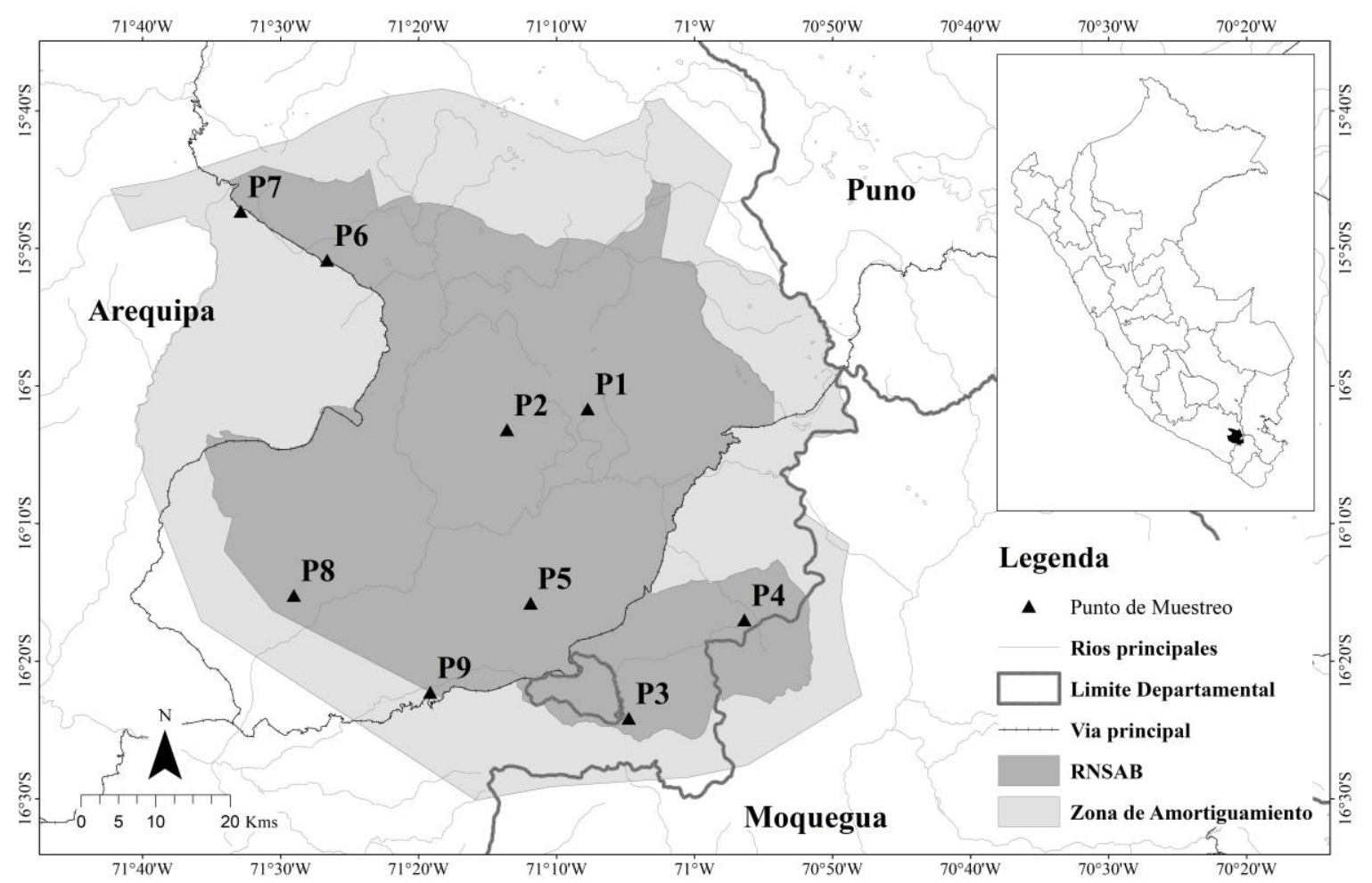

Figura 1. Mapa de ubicación de los puntos de muestreo evaluados en la Reserva Nacional Salinas y Aguada Blanca.

Location of sampling points assessed in Salinas y Aguada Blanca National Reserve.

dominado por Alchemilla diplophylla Diels y Distichia muscoides Nees et Meyen (11.085,31 ha); Yaretal, dominado por Azorella spp. Lamarck y Pycnophyllum spp. J. Rémy (8.146,16 ha); y queñual, dominado por Polylepis rugulosa Bitter (4.295,48 ha) (Zeballos et al. 2010).

Muestreo. Los trabajos de campo se realizaron durante los meses de enero y febrero de 2017. Se ubicaron nueve puntos de muestreo en la RNSAB (cuadro 1), siguiendo las recomendaciones de MINAM (2015) y diseños de muestreo de estudios previos (Crispin 2015, Morales 2015, Limache 2016, Flores 2017, Mango 2017, Sarcca 2017). A continuación, se detalla las formaciones vegetales evaluadas:

- Pajonal. Se instalaron seis parcelas de $3 * 3 \mathrm{~m}$, en las cuales se colectó la totalidad de plántulas de los géneros Stipa y/o Festuca. La parte aérea y las raíces fueron pesadas en fresco y luego empaquetadas en bolsas de papel para su traslado al laboratorio. Se pesó un total de $75,757 \mathrm{~kg}$ de plántulas frescas, las cuales presentaron en promedio $66,8 \%$ de materia seca.

- Tolar. Se instalaron seis parcelas de $50 * 20 \mathrm{~m}$, en las cuales se realizó el conteo total de plántulas de los géneros Parastrephia y/o Baccharis. Luego, se colectó al azar una muestra de 10 plántulas (parte aérea y raíces) por parcela, las cuales fueron pesadas en fresco y luego empaquetadas para su traslado al laboratorio. Se pesó $35,126 \mathrm{~kg}$ de plántulas frescas, provenientes de 60 individuos, los cuales presentaron en promedio 59,71\% de materia seca; además se registró una densidad promedio de 8.085 individuos/ha.

- Bofedal. Se instalaron seis parcelas de $1^{*} 1 \mathrm{~m}$, cada una de las cuales fue dividida en 4 sub-parcelas de $50 * 50 \mathrm{~cm}$ y ubicadas en forma cruz, a una distancia de $7 \mathrm{~m}$ desde el punto central de la parcela. $\mathrm{La}$ parte aérea y las raíces, hasta una profundidad de $30 \mathrm{~cm}$, fueron colectadas, pesadas en fresco, y luego empaquetadas para su traslado al laboratorio. Se pesó un total de $1.189,446 \mathrm{~kg}$ de muestra fresca, presentando en promedio 17,21\% de materia seca.

- Yaretal. Se instalaron seis parcelas de 50*20 m, en las cuales se realizó un conteo total de plántulas del género Azorella. Luego, se escogió al azar seis plántulas por parcela para estimar el volumen de su parte aérea, en función a su altura, diámetro mayor y diámetro menor. Finalmente, en las mismas plántulas medidas se colectó una sub-muestra de volumen conocido (prisma rectangular de $7 \mathrm{~cm}$ de lado y " $z$ " de profundidad), la cual fue pesada en fresco y luego empaquetadas para su traslado al laboratorio. Se pesó 33,609 kg de muestra 
fresca, provenientes de 36 individuos, los cuales presentaron en promedio $43,62 \%$ de materia seca y densidad promedio de 981,7 individuos/ha.

- Queñual. Se instalaron cuatro parcelas de 50*20 m, en las cuales se midió la totalidad de plántulas de Polylepis rugulosa. Se obtuvo la altura total y el diámetro de la copa para cada individuo, información necesaria para aplicar la fórmula dasométrica propuesta Sarcca (2017) para P. rugulosa. Se midió 206 individuos y se registró una densidad promedio de 515 individuos/ha.

Carbono almacenado. Se utilizó el método calorimétrico para obtener el carbono almacenado en las muestras (Eduarte y Segura 1998). Para ello, las muestras colectadas en las parcelas de Pajonal, Tolar, Bofedal y Yaretal fueron desecadas sobre un plástico negro expuesto a la radiación solar, por períodos de tres horas a $62{ }^{\circ} \mathrm{C}$, durante cinco a seis días consecutivos. Luego, dichas muestras fueron llevadas al laboratorio para terminar el proceso a $80{ }^{\circ} \mathrm{C}$ durante $24 \mathrm{~h}$, hasta alcanzar un peso seco constante, valor conocido también como Biomasa. En el caso del Yaretal, la biomasa contenida en cada submuestra fue extrapolada al volumen total de cada plántula. En el caso del Queñoal, la biomasa fue estimada con la fórmula dasométrica propuesta por Sarcca (2017) [1]:

Donde:

$$
\mathrm{Bio}=0,16496[\mathrm{~A}+\mathrm{D}] 2,667785
$$

Bio $=$ Biomasa $(\mathrm{kg})$

$\mathrm{A}=$ altura total de la plántula $(\mathrm{m})$

$\mathrm{D}=$ diámetro de la copa $(\mathrm{m})$

La cantidad de carbono almacenado en las parcelas evaluadas fue estimada con la fórmula [2]. En el caso del Tolar, el valor de carbono almacenado contenido en las 10 plántulas colectadas en cada parcela fue extrapolado con la densidad de plántulas contabilizadas en la misma parcela. De forma similar, en el Yaretal, el valor de carbono almacenado contenido en las seis plántulas medidas en cada parcela fue extrapolado a la densidad de plántulas contabilizadas en la misma parcela.

Donde:

$$
\mathrm{CA}=\mathrm{Bio} * \mathrm{FC}
$$

$\mathrm{CA}=$ Carbono almacenado

Bio $=$ Biomasa $(\mathrm{Mg})$

$\mathrm{FC}=$ Factor de carbono en biomasa $(0,5)$, siguiendo a Eggleston et al. (2006).

Carbono capturado (CC). La cantidad de carbono capturado en cada parcela fue estimada con la fórmula [3]:

Donde:

$$
\mathrm{CC}=\mathrm{CA} * \mathrm{Kr}
$$

$\mathrm{CC}=$ Carbono capturado

$\mathrm{CA}=$ Carbono almacenado

$\mathrm{Kr}=$ Factor de conversión de carbono a $\mathrm{CO}_{2}(3,66)$, siguiendo a Eggleston et al. (2006).

Valoración económica. A pesar de que el Perú no aplica un impuesto al carbono nacional, se aplicó el precio de mercado voluntario de USD 6,39 por tonelada de $\mathrm{CO}_{2}$ equivalente, estimado a partir de la evaluación de la rentabilidad social de proyectos de inversión pública llevados a cabo en el territorio peruano en el año 2018 y 2019 (Trinidad y Ortiz 2019). Dicho monto fue multiplicado a la cantidad promedio de $\mathrm{CO}_{2}$ capturado en las parcelas evaluadas y posteriormente, el valor obtenido fue extrapolado a toda el área de la RNSAB con apoyo de cartografía disponible para las formaciones vegetales presentes en la Reserva (Zeballos et al. 2010).

\begin{tabular}{|c|c|c|c|c|c|}
\hline \multirow{2}{*}{$\begin{array}{l}\text { Puntos de } \\
\text { Muestreo }\end{array}$} & \multirow{2}{*}{$\begin{array}{l}\text { Localidad de } \\
\text { Referencia }\end{array}$} & \multirow{2}{*}{$\begin{array}{c}\text { Unidades } \\
\text { de vegetación }\end{array}$} & \multicolumn{3}{|c|}{ Coordenadas geográficas } \\
\hline & & & $\mathrm{S}$ & $\mathrm{W}$ & m s.n.m \\
\hline $\mathrm{P} 1$ & Condori & Pajonal, Tolar & $16^{\circ} 01,652^{\prime}$ & $71^{\circ} 07,746^{\prime}$ & 4.353 \\
\hline $\mathrm{P} 2$ & Huayllacucho & Tolar & $16^{\circ} 03,179^{\prime}$ & $71^{\circ} 13,597^{\prime}$ & 4.460 \\
\hline P3 & Santa Lucia de Salinas & Pajonal, Bofedal & $16^{\circ} 24,145^{\prime}$ & $71^{\circ} 04,781^{\prime}$ & 4.322 \\
\hline P4 & Vía Cancosani & Bofedal, Yaretal & $16^{\circ} 16,998^{\prime}$ & $70^{\circ} 56,390^{\prime}$ & 4.425 \\
\hline P5 & Salinas Huito - Cerro Pucasaya & Tolar, Yaretal & $16^{\circ} 15,783^{\prime}$ & $71^{\circ} 11,892^{\prime}$ & 4.987 \\
\hline P6 & Pampas de Tocra & Pajonal, Bofedal & $15^{\circ} 50,851^{\prime}$ & $71^{\circ} 26,635^{\prime}$ & 4.344 \\
\hline P7 & Cerro Chucura & Yaretal & $15^{\circ} 47,299^{\prime}$ & $71^{\circ} 32,891^{\prime}$ & 4.642 \\
\hline P8 & Pampa Cañahuas - Cabrerías & Pajonal, Queñual & $16^{\circ} 15,219^{\prime}$ & $71^{\circ} 29,053^{\prime}$ & 4.727 \\
\hline P9 & El Simbral & Queñual & $16^{\circ} 22,264^{\prime}$ & $71^{\circ} 19,160^{\prime}$ & 3.810 \\
\hline
\end{tabular}

Cuadro 1. Puntos de muestreo ubicados en la Reserva Nacional Salinas y Aguada Blanca.

Sampling points located in Salinas y Aguada Blanca National Reserve. 
Finalmente, el valor económico total asociado al secuestro y almacenamiento de $\mathrm{CO}_{2}$ en la RNSAB fue calculada por la suma de los valores económicos obtenidos para el Pajonal, Tolar, Bofedal, Yaretal y Queñual.

\section{RESULTADOS}

Los resultados muestran que el servicio de secuestro y almacenamiento de $\mathrm{CO}_{2}$ fluctúa según el tipo de formación vegetal y el área que esta ocupa en la Reserva Nacional de Salinas y Aguada Blanca (cuadro 2).

Respecto a la cantidad de carbono almacenado, se observó una fluctuación de 4,66 a 639,39 Mg/ha de $\mathrm{CO}_{2}$ en el área de estudio, siendo el Bofedal la formación vegetal donde se concentró la mayor cantidad de carbono almacenado $\left(639,39 \mathrm{Mg} / \mathrm{ha}\right.$ de $\left.\mathrm{CO}_{2}\right)$, seguido del Yaretal $(98,63)$, Pajonal $(16,84)$, Queñual $(11,6)$ y Tolar $(4,66)$. Dichos valores al ser extrapolados a la superficie total de cada formación vegetal en el área de estudio, mostró que la RNSAB resguarda al menos $13.507 .104,16$ toneladas métricas de $\mathrm{CO}_{2}$ equivalente.

Respecto al valor económico del servicio de secuestro y almacenamiento de $\mathrm{CO}_{2}$ por formación vegetal, se observó una fluctuación de U\$D 318.350,12 a U\$D 45.291.274,34, siendo el Bofedal la formación vegetal donde se obtuvo el mayor valor económico (USD 45.291.274,34), seguido del Pajonal (34.119.364,04), Yaretal $(5.133 .964,15)$, Tolar $(1.447 .442,93)$ y Queñual $(318.350,12)$. El valor económico del servicio de secuestro y almacenamiento de $\mathrm{CO}_{2}$ en la RNSAB fue estimado en U\$D 86.310.395,58.

\section{DISCUSIÓN}

Los resultados confirman la gran importancia que tiene la vegetación alto-andina en cuanto al servicio ecosistémico de secuestro y almacenamiento de $\mathrm{CO}_{2}$, en función al tipo de formación vegetal y su extensión.

Cuadro 2. Diseño de muestreo y estimación del valor económico del servicio ecosistémico de secuestro y almacenamiento de $\mathrm{CO}_{2}$ en la Reserva Nacional Salinas y Aguada Blanca. National Reserve.

Sampling design and estimation of economic value of the ecosystem service of carbon capture and storage in Salinas y Aguada Blanca

\begin{tabular}{|c|c|c|c|c|c|}
\hline Característica & Pajonal & Tolar & Bofedal & Yaretal & Queñual \\
\hline $\begin{array}{l}\text { Dimensiones de parcelas } \\
\text { evaluadas }(\mathrm{m})\end{array}$ & $3 * 3$ & $50 * 20$ & $1 * 1$ & $50 * 20$ & $50 * 20$ \\
\hline $\begin{array}{l}\text { Densidad de plántulas, promedio } \\
\pm \mathrm{SD} \text { (individuos/parcela) }\end{array}$ & - & $\begin{array}{c}808,50 \\
\pm \\
338,90\end{array}$ & - & $\begin{array}{l}98,17 \\
\quad \pm \\
23,06\end{array}$ & $\begin{array}{c}51,5 \\
\pm \\
23,13\end{array}$ \\
\hline Área de parcelas evaluadas (ha) & 0,0009 & 0,1 & 0,0001 & 0,1 & 0,1 \\
\hline Réplicas (n) & 6 & 6 & 6 & 6 & 4 \\
\hline $\begin{array}{l}\mathrm{CO}_{2} \text { capturado, promedio } \pm \mathrm{SD} \\
(\mathrm{Mg} / \text { parcela })\end{array}$ & $\begin{array}{c}0,015153 \\
\pm \\
0,004889\end{array}$ & $\begin{array}{c}0,465856 \\
\pm \\
0,237645\end{array}$ & $\begin{array}{c}0,063939 \\
\pm \\
0,027761\end{array}$ & $\begin{array}{c}9,862773 \\
\pm \\
10,13338\end{array}$ & $\begin{array}{c}1,159825 \\
\pm \\
0,610465\end{array}$ \\
\hline $\begin{array}{l}\mathrm{CO}_{2} \text { capturado, promedio } \pm \mathrm{SD} \\
(\mathrm{Mg} / \mathrm{ha})\end{array}$ & $\begin{array}{c}16,837 \\
\pm \\
5,432\end{array}$ & $\begin{array}{l}4,659 \\
\quad \pm \\
2,376\end{array}$ & $\begin{array}{c}639,39 \\
\pm \\
277,610\end{array}$ & $\begin{array}{c}98,62773 \\
\pm \\
101,334\end{array}$ & $\begin{array}{c}11,59825 \\
\pm \\
6,105\end{array}$ \\
\hline $\begin{array}{l}\mathrm{CO}_{2} \text { capturado, promedio } \pm \\
\mathrm{SD}\left(\mathrm{Mg} / \mathrm{km}^{2}\right)\end{array}$ & $\begin{array}{c}1.683,667 \\
\pm \\
543,222\end{array}$ & $\begin{array}{c}465,856 \\
\pm \\
237,645\end{array}$ & $\begin{array}{c}63.939,340 \\
\pm \\
27.759,650\end{array}$ & $\begin{array}{c}9.862,773 \\
\pm \\
10.133,38\end{array}$ & $\begin{array}{c}1.159,825 \\
\pm \\
610,465\end{array}$ \\
\hline $\begin{array}{l}\text { Área total de la Unidad de } \\
\text { Vegetación (ha) en la RNSAB }\end{array}$ & $317.134,84$ & $48.623,80$ & $11.085,31$ & $8.146,16$ & $4.295,48$ \\
\hline $\begin{array}{l}\mathrm{CO}_{2} \text { capturado por Unidad de } \\
\text { vegetación }(\mathrm{Mg}) \text { en la } \mathrm{RNSAB}\end{array}$ & $5.339 .493,59$ & $226.516,89$ & $7.087 .836,36$ & $803.437,27$ & $49.820,05$ \\
\hline Precio de tonelada de $\mathrm{CO}_{2}(\mathrm{U} \$ \mathrm{D})$ & 6,39 & 6,39 & 6,39 & 6,39 & 6,39 \\
\hline $\begin{array}{l}\text { Valor económico por Unidad de } \\
\text { vegetación (USD) en la RNSAB }\end{array}$ & $34.119 .364,04$ & $1.447 .442,93$ & $45.291 .274,34$ & $5.133 .964,15$ & $318.350,12$ \\
\hline $\begin{array}{l}\text { Valor económico del servicio } \\
\text { ecosistémico (USD) en la } \\
\text { RNSAB }\end{array}$ & & & $86.310 .395,58$ & & \\
\hline
\end{tabular}


Por un lado, la cantidad de carbono almacenado obtenida para el Bofedal $\left(639,39 \mathrm{Mg} / \mathrm{ha}\right.$ de $\left.\mathrm{CO}_{2}\right)$ fue mayor a lo reportado por Crispin (2015) con $518,8 \mathrm{Mg} / \mathrm{ha}$ de $\mathrm{CO}_{2}$, en Huancavelica, Perú (13 $19,779^{\prime}$ S, $74^{\circ} 58,902^{\prime}$ W); sin embargo fue menor a la cantidad registrada por Mango

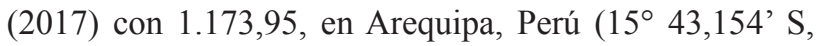
$\left.71^{\circ} 18,823^{\prime} \mathrm{W}\right)$. Dichas variaciones pueden ser consecuencia del manejo que realizan los pobladores locales en los bofedales para aumentar su productividad y sirvan de forraje para su ganado. Mientras que, la cantidad de carbono almacenado obtenida para el Pajonal $(16,84 \mathrm{Mg} /$ ha de $\mathrm{CO}_{2}$ ) fue relativamente similar a lo reportado por la FAO (2010) y Medrano et al. (2012), con valores de 16,5 y 15,43 , respectivamente; en cambio Gibbon et al. (2010) y Flores (2017) reportaron valores inferiores (7,5 a 10,78). Dichas variaciones pueden ser explicadas debido a que los pajonales evaluados en cada estudio estuvieron dominados por distintas especies de Poaceae (Stipa, Festuca y/o Deyeuxia) y por variaciones en el diseño de muestreo (Gibbon et al. 2010 solo evalúan biomasa aérea).

Aquí, se documenta por primera vez la cantidad de carbono almacenado para las formaciones vegetales de Yaretal $\left(98,63 \mathrm{Mg} / \mathrm{ha}\right.$ de $\left.\mathrm{CO}_{2}\right)$ y Tolar $(4,66)$, dejando una fuente de referencia para valorar su importancia como sumidero de carbono en futuras investigaciones.

La cantidad de carbono almacenado obtenida para el queñual $\left(11,6 \mathrm{Mg} / \mathrm{ha}\right.$ de $\left.\mathrm{CO}_{2}\right)$ fue menor a lo reportado por FAO (2010), con $56,3 \mathrm{Mg}$ /ha de $\mathrm{CO}_{2}$, y Vásquez et al. (2014), con 439; sin embargo, fue mayor a lo reportado por Glave y Pizarro (2001), Morales (2015) y Sarcca (2017), con valores de 3,55, 0,69 y 7,57, respectivamente. Dichas variaciones podrían ser explicadas a la especie y densidad de árboles en cada zona de estudio.

Cabe resaltar, que los bosques son particularmente importantes debido a que los árboles almacenan más carbono por unidad de área (en forma de madera) que otros tipos de vegetación (Houghton 2007), llegando a almacenar en algunos casos hasta $335,1 \mathrm{Mg} / \mathrm{ha}$ de $\mathrm{CO}_{2}$ en bosques húmedos tropicales de tierras bajas (Martel y Cairampoma 2012). Sin embargo, los resultados demuestran que el Bofedal puede almacenar una mayor cantidad de carbono $\left(639,39 \mathrm{Mg} / \mathrm{ha}\right.$ de $\left.\mathrm{CO}_{2}\right)$, ya que este utiliza otras estructuras como sumideros de carbono (raíces y/o masa muerta en el suelo). Un panorama similar fue observado por Castañeda-Martín y Montes-Pulido (2017) quienes reportaron un almacenamiento de 119 a $397 \mathrm{Mg} / \mathrm{ha}$ de $\mathrm{CO}_{2}$ en los primeros $40 \mathrm{~cm}$ de profundidad del suelo del Páramo.

Por otro lado, respecto al valor económico, a pesar de que el Bofedal está representado en una pequeña área de la superficie total de la RNSAB (2,58 \%), este aportó un poco más de la mitad del valor económico total de secuestro y almacenamiento de $\mathrm{CO}_{2}$ en la RNSAB (52,48 \%) (figura 2), corroborando su relevancia en la lucha contra la mitigación del cambio climático (Hribljan et al. 2015). El Pajonal se ubicó en segundo lugar de importancia de contribución al valor económico total del servicio de secuestro y alma-

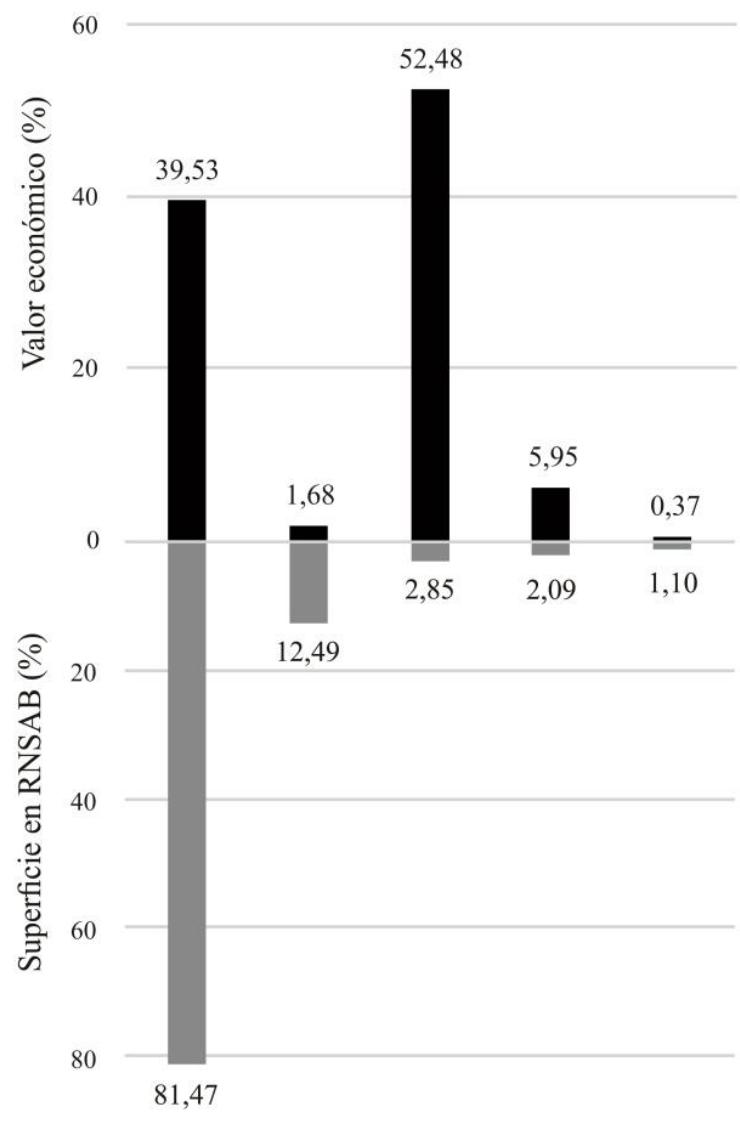

Pajonal Tolar Bofedal Yaretal Queñual

Figura 2. Relación entre el valor económico estimado y el área de cada formación vegetal en la Reserva Nacional Salinas y Aguada Blanca.

The relationship between economic value estimated and area of each vegetal formation in Salinas y Aguada Blanca National Reserve.

cenamiento de $\mathrm{CO}_{2}$, el cual se encuentra ampliamente representada en la superficie total de la RNSAB $(81,47 \%)$. Mientras que, el Yaretal, Tolar y Queñual fueron las formaciones vegetales que menos aportaron al valor económico total $(5,95 \%, 1,68 \%$ y $0,37 \%$, respectivamente), las cuales se encuentra poco representadas en la superficie total de la RNSAB.

En las últimas cuatro décadas, los departamentos de Apurímac, Arequipa, Cusco y Puno han agrupado el $80 \%$ de superficie afectada por fuego en la Región Andina del Perú (Manta et al. 2018). Un ejemplo de ello, son los recientes incendios forestales significativos provocados en la región de Arequipa (Andagua, Miraflores, Mariano Melgar, Pocsi, Yura, entre otros), los cuales ocasionaron la pérdida de pajonales, matorrales y queñuales, afectando la situación socioeconómica, desarrollo y bienestar de la población. Ante dicho panorama, se espera que el presente estudio sirva de marco de referencia en temas de concientización ambiental (conservación de la cobertura vegetal), 
elaboración de instrumentos de gestión ambiental (diseño e implementación de sanciones por destrucción de la cobertura vegetal), contabilidad nacional, entre otros., con miras hacia una adecuada gestión ambiental de los ecosistemas desérticos de la puna seca del suroeste del Perú.

A nivel de la región de Arequipa, solo se conoce dos estudios relacionados a la valoración económica de servicios ambientales proporcionados por la RNSAB. El primero, estimó un valor económico de U\$D 2.132.502,57 por el servicio de provisión de agua para Arequipa Metropolitana (Loyola 2007); mientras que el segundo, estimó un valor económico de U\$D 384.209,60 por concepto de aprovechamiento de fibra de vicuña (Medina et al. 2018). Siguiendo dichas iniciativas, se insta el desarrollo de investigaciones que involucren la valoración de otros bienes y servicios ecosistémicos que brinda la RNSAB, tales como: ecoturismo, fijación de nitrógeno atmosférico, medicina tradicional, entre otros.

\section{CONCLUSIONES}

El valor económico de secuestro y almacenamiento de carbono por la vegetación de la RNSAB asciende a U\$D 86.310.395,58, siendo el Bofedal quien aportó el 52,48 \% de dicho valor, seguido del Pajonal (39,53\%), Yaretal $(5,95 \%)$, Tolar $(1,68 \%)$ y Queñual $(0,37 \%)$.

Considerando la importancia relativa del Bofedal, $\mathrm{Pa}$ jonal, Yaretal, Tolar y Queñual como sumideros de carbono en Los Andes, se recomienda la implementación de un programa de monitoreo a largo plazo para evaluar las fluctuaciones del secuestro y almacenamiento de carbono en la puna seca del suroeste del Perú.

\section{AGRADECIMIENTOS}

Se agradece a Edgardo Medina y Rosa Pacheco por el apoyo logístico en los trabajos de campo, y al Dr. Evaristo López por el apoyo en laboratorio. Se agradece a la Universidad Nacional de San Agustín de Arequipa (UNSA) por el financiamiento (Contrato de Subvención $\mathrm{N}^{\circ} \mathrm{TM}-$ 0005-2016), habiendo sido ejecutado con el apoyo en la gestión de CIENCIACTIVA y CONCYTEC, y al Servicio Nacional de Áreas Naturales Protegidas (SERNANP) por facilitar la autorización de investigación dentro de la Reserva Nacional de Salinas y Aguada Blanca (RNSAB).

\section{REFERENCIAS}

Castañeda-Martín AE, CR Montes-Pulido. 2017. Carbono almacenado en páramo andino. Entramado 13(1): 210-221. DOI: $10.18041 /$ entramado.2017v13n1.25112

Crispin M. 2015. Valoración económica ambiental de los Bofedales del distrito de Pilpichaca, Huancavelica, Perú. Tesis de Maestría en Ciencias Ambientales. Lima, Perú. Escuela de Posgrado, Universidad Nacional Agraria La Molina. 154 p.

Eduarte E, M Segura. 1998. Determinación de carbón utilizando la calorimetría. Revista de Ciencias Ambientales 15(2): 54-
55. DOI: $10.15359 /$ rca.15-1.6

Eggleston S, L Buendia, K Miwa, T Ngara, K Tanabe. 2006. 2006 IPCC Guidelines for National Greenhouse Gas Inventories, Volume 4: agriculture, forestry, and other land use. Intergovernmental Panel on Climate Change (IPCC). Kanagawa, Japan. Institute for Global Environmental Strategies-IPCC. $627 \mathrm{p}$.

FAO (Organización de las Naciones Unidas para la Agricultura y la Alimentación, IT). 2010. Evaluación de los recursos forestales mundiales 2010, informe principal. Estudio FAO Montes 163. Roma, Italia. Organización de las Naciones Unidas para la Agricultura y la Alimentación. 346 p.

Flores M. 2017. Captura de Dióxido de Carbono $\left(\mathrm{CO}_{2}\right)$ en la "Chillihua" (Festuca dolichophylla Presl) de los pastizales del Cipillpa-Puno. Tesis de Ingeniero Agrónomo. Puno, Perú. Facultad de Ciencias Agrarias, Universidad Nacional del Altiplano. 119 p.

Glave M, R Pizarro. 2001. Valoración Económica de la Diversidad Biológica y Servicios Ambientales en el Perú. Lima, Perú. Proyecto BIOFOR. INRENA/IRG/USAID. 473 p.

Gibbon A, MR Silman, Y Malhi, JB Fisher, P Meir, M Zimmermann, GC Dargie, WR Farfan, KC Garcia. 2010. Ecosystem carbon storage across the grassland-forest transition in the high Andes of Manu National Park, Peru. Ecosystems 13: 1097-1111. DOI: 10.1007/s10021-010-9376-8

Hribljan JA, DJ Cooper, J Sueltenfuss, EC Wolf, KA Heckman, EA Lilleskov, RA Chimner. 2015. Carbon storage and longterm rate of accumulation in high-altitude Andean peatlands of Bolivia. Mires and Peat 15(12): 1-12.

Houghton RA. 2007. Balancing the global carbon budget. Annual Review of Earth and Planetary Sciences 35(1): 313-347. DOI: 10.1146/annurev.earth.35.031306.140057

Limache IV. 2016. Valorización económica ambiental del ecosistema de Bofedales en el centro poblado de Huaytire - Tacna. Tesis de Maestría en Gestión Ambiental y Desarrollo Sostenible. Tacna, Perú. Escuela de Posgrado, Universidad Nacional Jorge Basadre Grohmann. 209 p.

Loyola R. 2007. Valoración del Servicio Ambiental de Provisión de Agua con Base en la Reserva Nacional Salinas y Aguada Blanca - Cuenca del Río Chili. Lima, Perú. PROFONANPE. 228 p.

Mango BC. 2017. Valoración económica de los servicios ecosistémicos de regulación, de los bofedales del centro poblado de Chalhuanca, distrito de Yanque, provincia de Caylloma, región Arequipa. Tesis de Economista. Arequipa, Perú. Facultad de Economía, Universidad Nacional de San Agustín de Arequipa. 96 p.

Manta MI, R Kometter, A Navia. 2018. Evaluation of wildfire danger in the Peruvian Andes: First step for its reduction and adaptation. In Viegas DX ed. Advances in Forest Fire Research. Imprensa da Universidade de Coimbra. Coimbra, Portugal. p. 44-56.

Martel C, L Cairampoma. 2012. Cuantificación del carbono almacenado en formaciones vegetales amazónicas en "CICRA”, Madre de Dios (Perú). Ecología Aplicada 11(2): 59-65. DOI: $10.21704 /$ rea.v11i1-2.426

MINAM. 2015. Guía de Inventario de la Flora y Vegetación. Dirección General de Evaluación, Valoración y Financiamiento del Patrimonio Natural, Ministerio del Ambiente (R.M. No 059-2015-MINAM). Lima, Perú. 49 p.

Medina CE, YK Medina, EF Bocardo. 2018. Valor económico de 
la fibra de vicuña en la Reserva Nacional Salinas y Aguada Blanca (Arequipa, Perú). Idesia 36(4). DOI: $10.4067 /$ $\underline{\mathrm{S} 0718-34292018005002002}$

Medrano R, L Chupan, M Vila. 2012. Almacenamiento de carbono en especies predominantes de flora en el Lago Chinchaycocha. Apuntes de ciencia \& sociedad 2(2): 110-117. DOI: $10.18259 /$ acs. 2012013

Morales FT. 2015. Estado actual del bosque de Polylepis y su eficiencia en la captura de CO, en la provincia Tarata, departamento de Tacna. Ciencia y Desarrollo 19: 36-43. DOI: $10.33326 / 26176033.2015 .19 .479$

Phillips OL, RJW Brienen, RAINFOR. 2017. Carbon uptake by mature Amazon forests has mitigated Amazon nations' carbon emissions. Carbon Balance Manage 12: 1. DOI: 10.1186/s13021-016-0069-2

Sarcca YR. 2017. Valoración económica del servicio ecosistémico de secuestro y almacenamiento de carbono en el bosque de Polylepis del Pichu pichu, Arequipa- 2016. Tesis de Ingeniero Ambiental. Arequipa, Perú, Facultad de Ingeniería de Procesos, Universidad Nacional de San Agustín de Arequipa. $152 \mathrm{p}$.

Sullivan MJP, J Talbot, SL Lewis, OL Phillips, L Qie, SK Begne, J Chaves, A Cuni-Sanchez, W Hubau, G Lopez-Gonzalez, L Miles, A Monteagudo-Mendoza, B Sonké, T Sunderland, H Steege, LJT White, K Affum-Baffoe. 2017. Diversity and carbon storage across the tropical forest biome. Scientific Reports 7: 39102. DOI: 10.5521/FORESTPLOTS.NET/2016 3

Trinidad C, E Ortiz. 2019. Precio al carbono en el Perú: transición energética y justicia climática. In Trinidad $\mathrm{C}$ ed. Precio al carbono en América Latina Tendencias y oportunidades. Lima, Perú. Sociedad Peruana de Derecho Ambiental y Fundación Konrad Adenauer. p. 270-319.

Vásquez E, B Ladd, N Borchard. 2014. Carbon storage in a highaltitude Polylepis woodland in the Peruvian Andes. Alpine Botany 124(1): 71-75. DOI:10.1007/s00035-014-0126-y

Zeballos H, JA Ochoa, E López. 2010. Diversidad Biológica de la Reserva Nacional de Salinas y Aguada Blanca. Arequipa, Perú. DESCO, INRENA, PROFONANPE. 313 p.

Recibido: 23/01/20

Aceptado: 11/05/20 